Ann. Zootech., 1977, 26 (4), 565.57.4.

\title{
Influence de trois niveaux d'alimentation de lapines futures reproductrices sur l'ardeur sexuelle et la fertilité à 4 mois
}

Lea VAN DEN BROF,CK et Ph. LAMPO

avec la collaboration technique de $\mathrm{H}$. Denoo

Chaire de Génétique animale et d'Elevage, Faculté de Médecine vétévinaire. Rijksuniversiteit, Gent Heidestraat, I9. B. g220 Mevelbeke, Belgique

\section{Résumé}

Trois niveaux d'alimentation pour futures reproductrices ont été étudiés sur trois groupes de 25 lapines. Le premier accouplement fut essayé à l'âge de 4 mois. Le rationnement sévère $(\mathrm{I} 5 \mathrm{O} \mathrm{g} / \mathrm{j})$ est rejeté, le rationnement combiné avec une période d'alimentation libre avant le preInier accouplement semble économique, mais les meilleurs résultats ont été obtenus avec les lapines nourries à volonté en permanence.

\section{Introduction}

Quelque temps après la mise en place d'un contrôle de gestation par palpation dans un cheptel existant, par lequel nous espérions accélérer la reproduction et économiser de l'aliment, nous avons éprouvé de grandes difficultés à mettre en reproduction les lapines nullipares. Les lapines pluripares par contre, accouplées aux mêmes mâles, et élevées dans les mêmes conditions ne présentaient aucune difficulté. Nous avons constaté que les jeunes lapines étaient très agitées et affamées. Il nous a semblé possible que la restriction alimentaire à $150 \mathrm{~g}$ par jour, soit trop sévère pour les futures reproductrices. En effet, avant la mise en place du contrôle de gestation, ce rationnement était interrompu par six ou sept jours d'alimentation libre au moment où la mise-bas devait avoir lieu. Cette période pourrait être suffisante pour que les lapines puissent se "rattraper ". Ces jeunes lapines "infertiles ", qui, en outre n'acceptaient pas les saillies, furent alors alimentées à volonté pendant 4 jours; après cette période, elles s'accouplèrent sans 
difficulté, et beaucoup d'entre elles furent gestantes. Ces observations nous ont donné l'impression que la reproduction de nos lapines pouvait être entravée par une alimentation insuffisante, et que les jeunes lapines pouvaient se "rattraper" par un "flushing "de quelques jours. Nous nous sommes donc proposés d'étudier l'effet d'un flushing sur des lapines rationnées depuis 1'âge de $70-77$ jours, en comparaison avec un rationnement continu d'une part, et avec une alimentation à volonté d'autre part.

\section{Bibliographie}

Il n'y a pas encore d'unanimité à propos des besoins nutritifs de futurs reproducteurs. LEROY (I949) propose un besoin énergétique pour l'entretien de 74 à $96 \mathrm{~g}$ TDN par jour pour des lapines de 3 à $4 \mathrm{~kg}$. De plus, pour un gain de poids de I $g$ à l'âge de 90 à $\Upsilon 50$ jours, ils auraient besoin de $\mathrm{I}, 5 \mathrm{~g}$ TDN. Ces futurs reproducteurs de 3 à $4 \mathrm{~kg}$ avec un gain moyen de $33,3 \mathrm{~g}$ par jour aturaient donc besoin d'environ I $35 \mathrm{~g}$ de TDN, soit de $225 \mathrm{~g}$ d'un aliment commercial contenant à peu près 60 p. cent de TDN. AXELSON et ERIKSON (I953) estiment les besoins énergétiques d'entretien d'un lapin de $3 \mathrm{~kg}$ à 200 Kcal d'énergie métabolisable; soit $74 \mathrm{~g}$ d'un aliment titrant $2700 \mathrm{Kcal} \mathrm{E} . \mathrm{M}$. $/ \mathrm{kg}$. Il faut y ajouter l'énergie nécessaire pour un gain de poids d'environ $33 \mathrm{~g}$ par jour.

Le N.R.C. (I966) situe les besoins nutritifs d'un futur reproducteur encore en croissance à $5,8 \mathrm{p}$. cent de son poids vif en matière sèche, à condition que la teneur en TDN atteigne 60 p. cent, et celle des protéines digestibles I I p. cent. Cela nous conduit de nouveau à 195 à $260 \mathrm{~g}$ de granulés par jour, pour des animaux de 3 à $4 \mathrm{~kg}$.

D'autre part, plus récemment, des expérimentateurs ont noté la consommation spontanée de lapins sélectionnés pour l'élevage rationnel: PRUD'HoN et al. (I975) ont enregistré des consommations de r94 à I60 g par jour à cet âge. Ces consommations sont confirmées par d'autres auteurs comme LEBAS (I975C) et LAPLACE et LEBAS (I975) avec un aliment titrant environ 2700 Kcal de E.M. Les conseils pratiques tiennent compte du risque d'engraissement des lapines nullipares, qui influencerait négativement les résultats de reproduction. La plupart des auteurs conseillent un rationnement des lapines dès l'âge de Io à I2 semaines. La ration ne devrait pas dépasser I50 g par jour, lorsque le niveau énergétique de l'aliment fourni atteint $2700 \mathrm{Kcal} \mathrm{E.M./kg} \mathrm{(LEBAS,} \mathrm{I975a;} \mathrm{OKERMAN,} \mathrm{I975).}$ Ces plans de rationnement sont appliqués dans des élevages rationnels ainsi que dans les laboratoires de recherche. Nulle part on ne trouve mention de phénomène de sous-alimentation. Cependant, nous n'avons pas pu retrouver des résultats concernant la première portée des lapines. Pour des études de production laitière, de fertilité, et de niveau d'alimentation durant la gestation, on préfère se servir de lapines ayant prouvé leur aptitude pour la reproduction (LEBAs et LAPLACF, I974, LEBAS, I975b). Pourtant, pour d'autres espèces d'animaux, on a démontré l'influence nocive d'une sous-alimentation durant la croissance sur les performances de reproduction. Le problème est très bien connu chez la truie nullipare (CASIDA, i963; ÉtTenne et Henry, I973; ÉtTienne et Dú́e, i 973; Goode, Warnick et Wallace, I965; LODGE et Mic Pherson, I96I), chez la brebis (CASIDA, I963), chez le "cotton-tail Rabbit "(KIRCKPATRICK et KIBBE, I97I) ainsi que chez le rat et la souris (LeATHEM, ig66; Short, Peters et Casida, ig68). 


\section{Matériel et méthodes}

\section{a) Bâtiment.}

Dans un bâtiment composé de panneaux sandwich industriels, au toit isolé en laine de verre, et au sol bétonné, se trouvent les batteries à trois étages, pourvues d'abreuvoirs automatiques à clapet (LUBING) alimentés en eau potable. La ventilation se fait par dépression, 1'air étant évacué par le toit. L'éclairage est assuré $\mathrm{I} 7 \mathrm{~h} / 24 \mathrm{~h}$ à l'aide de lampes TL. Un chauffage au gaz à air pulsé maintient la température au-dessus de $18^{\circ} \mathrm{C}$.

\section{b) Animaux et élevage}

Vingt-cinq trios de sœurs ont été sélectionnés à l'âge de $70-77$ jours, à un poids minimum de $2,50 \mathrm{~kg}$; I I trios appartiennent à la race Blanc de Termonde, les lapines de huit trios sont des Néo-Zélandaises blanches, et celles des six derniers proviennent de croisements entre ces deux races. L'indice de consommation durant la période d'engraissement variait de 2,4 à 4,8 ; en moyenne il était de 3,5 . Les laperaux, sevrés à 3I-36 jours, ont été alimentés à volonté durant 1'engraissement. Lorsque trois scurs d'une même portée atteignent le poids minimum à 1'âge prévu elles sont utilisées pour l'expérimentation. Elles sont installées dans des cages individuelles, dans le même bâtiment que les reproductrices. L.es femelles sont présentées au mâle dès l'âge de 4 mois, deux fois par semaine : le lundi et le vendredi, jusqu'à leur saillie. Une lapine refusant un mâle pendant plus de cinq minutes, est présentée immédiatement à un autre mâle et observée pendant au moins dix minutes. Nous avons une quinzaine de mâles adultes pour servir un cheptel de cent-vingt femelles, y compris les futures reproductrices. Le contrôle de gestation est effectué le lundi matin, I4 à I7 jours après la saillie, par un vétérinaire expérimenté. Une lapine est éliminée après cinq essais négatifs ou après deux saillies infécondes.

\section{c) Alimentation}

L'aliment distribué avait la composition suivante (d'après trois analyses en laboratoire) : matière sèche : $9 \mathrm{I} \mathrm{p}$. cent, matière azotée : $\mathrm{I} 8,5 \mathrm{p}$. cent; matières grasses : 4,I6 p. cent; extractif non azoté 46,95 p. cent, et cellulose brute : II,53. En fonction des coefficients de digestibilité, et de la teneur en énergie de différents composants d'une ration (Schieman et al., I97I) l'énergie métabolisable serait de $2733 \mathrm{Kcal} / \mathrm{kg}$. Une ration de ${ }_{5} 0 \mathrm{~g}$ représente alors 4 IO Kcal d'E.M. Le groupe de lapines rationnées " $\mathrm{R}$ ", reçoit $\mathrm{I}_{5} \mathrm{O} g$ de granulés par jour jusqu'à la mise-bas. Cette quantité est distribuée l'après-midi. Toutefois le vendredi elles reçoivent 3 rations pour le week-end.

Les lapines du groupe Flushing, " F », reçoivent la même ration, excepté pendant les 4 jours précédant le premier accouplement et jusqu'au jour de Ire saillie effective où elles sont nourries à volonté. En cas de palpation négative, le "flushing " est renouvelé jusqu'à une deuxième saillie. Enfin les lapines du groupe " $A$ " reçoivent chaque jour une quantité d'aliment supérieure à celle qu'elles feuvent consommer. 


\section{d) Statistique}

Pour les calculs statistiques nous avons utilisé l'analyse de variance, le test Duncan-Kramer et le test chi-carré. Les observations ont eu lieu de septembre I974 à juillet 1976 .

\section{Résultats}

\section{I. - Consommation d'aliment}

La consommation spontanée des lapines "A " pendant les trente jours qui précèdent le premier accouplement, est en moyenne de $300 \mathrm{~g}$ par jour. Elle se limite à $255 \mathrm{~g}$ par jour durant la gestation de ces mêmes lapines. Il n'y a pas de gaspillage important, grâce entre autre à une distribution quotidienne du granulé. Pendant les 4 premiers jours du flushing, les lapines " $F$ » ingèrent $345 \mathrm{~g}$ parjour. Cinq lapines " $F$ " non saillies le jour de la première présentation, ne consommaient plus etısuite que $285 \mathrm{~g}$ par jour jusqu'à leur saillie.

\section{2. - Poids des temelles}

Les différences de consommation se reflètent en premier lieu sur le poids des lapines à 4 mois, c'est-à-dire, le jour de la première présentation (tabl. I). Les poids des trois groupes diffèrent significativement. Au moment de la première saillie fécondante, le poids des lapines " $R$ " ne diffère plus du poids des lapines " $F$ ", ce qui laisse supposer que seules les lapines " $\mathrm{R}$ " les plus lourdes peuvent concevoir. Il est intéressant de remarquer que pendant la période d'engraissement ces lapines

\section{TABLEAU I}

Poids vif des lapines à 4 mois et au moment de la $I^{\mathrm{re}}$ saillie fécondante (en $g$ )

Weight of the does at an age of 4 months and at the moment of the first fortile mating $(g)$

\begin{tabular}{|c|c|c|c|c|c|c|}
\hline \multirow[b]{2}{*}{ Régime (feeding level). } & \multicolumn{3}{|c|}{$\begin{array}{c}4 \text { mois } \\
(4 \text { months })\end{array}$} & \multicolumn{3}{|c|}{$\begin{array}{l}{ }^{\mathrm{rc}} \text { saillie fécondante } \\
\text { (first fertile mating) }\end{array}$} \\
\hline & $n$ & $\mathrm{x}$ & $s_{\mathbf{X}}$ & $n$ & $\mathrm{x}$ & $s_{\mathbf{X}}$ \\
\hline \multicolumn{7}{|l|}{ Rationnement (vestricted feed- } \\
\hline$i n g) . . . . . . . .$. & 25 & $36 \mathrm{ro}$ & 250 & 9 & $385^{a}, a$ & 272 \\
\hline Flushing (Flushing) & 25 & $3910^{b}$ & 260 & 19 & $3969^{b}, d$ & 164 \\
\hline Ad libitum . . . . & 25 & $423^{c}$ & 322 & 24 & $427^{6 c}, e$ & 366 \\
\hline
\end{tabular}

Il n'y a pas de différence statistique entre les chiffres affectés de la même lettre. There is no signiflcant difference between figures dotted with the same letter. 


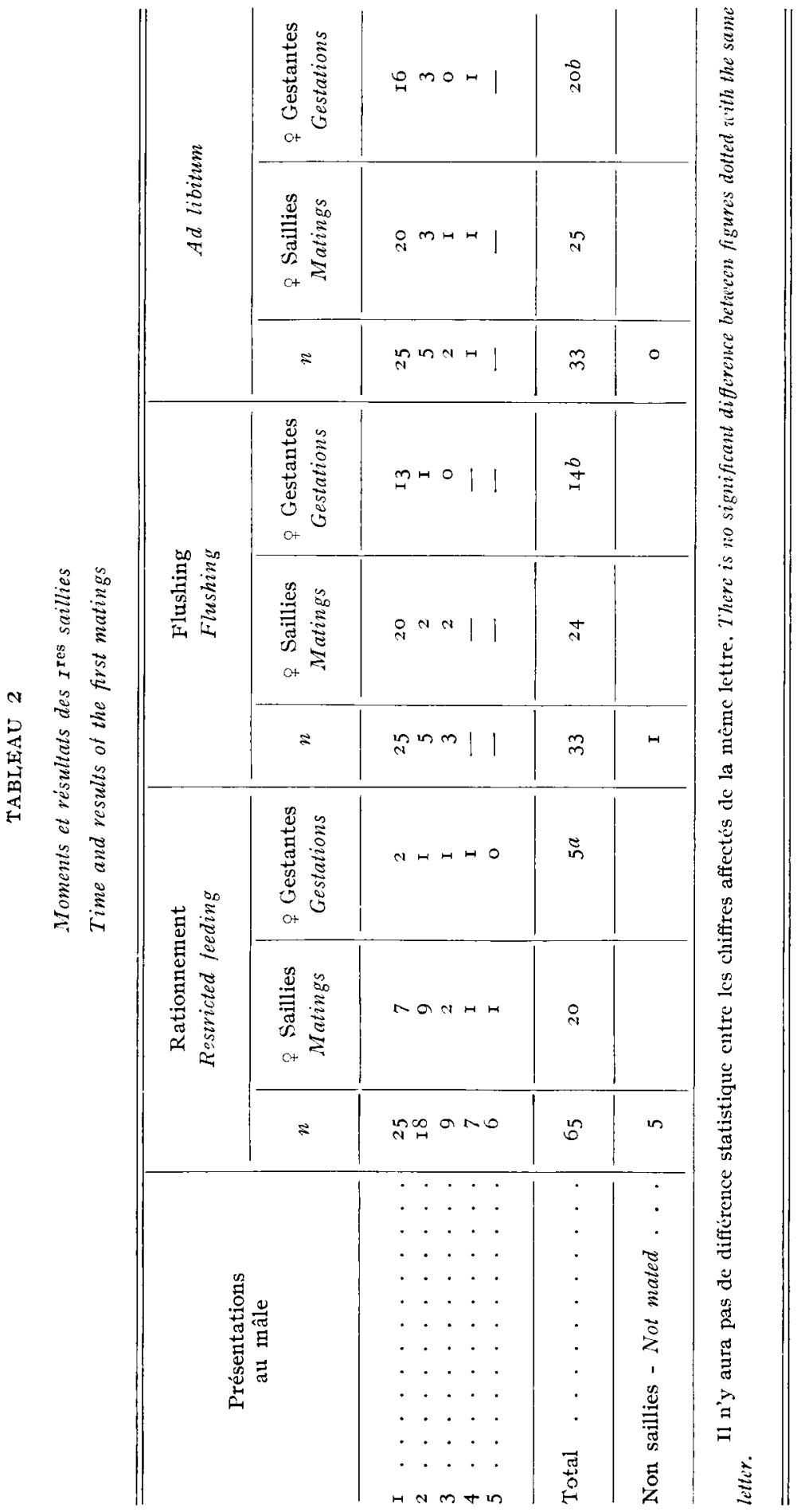


" $\mathrm{R}$ ) fécondes avaient eu un indice de consommation identique à celui obtenu en moyenne par les lapines " $\mathrm{R}$ " à savoir 3,5. La différence de poids entre les lapines " $\mathrm{F}$ » et les " $\mathrm{A}$ » reste significativement au moment de la première fécondation.

\section{3. - Acceptation du mâle et taux de gestation}

Le tableau 2 nous indique le nombre de présentations au mâle nécessaire pour obtenir une première saillie ainsi que le résultat de cette dernière. Il faut plus de présentations par lapine pour les femelles " $R$ ", et après cinq tentatives, $\mathrm{il}$ en reste encore 5 non saillies. Le taux de gestation (proportion gestation/saillie) est le même pour les lapines " $F$ " et " $A$ », tandis qu'il est nettement plus bas pour les " $R$ ". Lorsqu'on cumule les deux premières saillies, la deuxième n'étant effectuée que si la première avait été inefficace, on constate de nouveau (tab1. 3), que le taux de gestation est nettement plus faible pour les lapines rationnées, et ne diffère pas pour les 2 autres groupes. Ainsi par groupe de 25 femelles, on obtient plus de femelles gestantes pour renouveler le cheptel lorsqu'on les a nourries à volonté

TABLEAU 3

Comportement et poids vif des lapines

Behaviour and weight of the does

\begin{tabular}{|c|c|c|c|}
\hline & " $\mathrm{R}$ " & "F" & "A" \\
\hline Nombre de lapines présentées - Number of coupled does & 25 & 25 & 25 \\
\hline Nombre de lapines saillies - Number of mated does . . . & 20 & 24 & 25 \\
\hline Nombre de gestations - Number of pregnancies . . . . & $9^{a}$ & $19^{b}$ & $24^{c}$ \\
\hline Nombre total de présentations au mâle $-N$ umber of trials & 87 & 49 & 40 \\
\hline 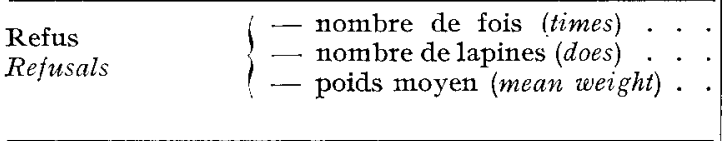 & $\begin{array}{l}59 \\
19 \\
3626 g d \\
\pm 269\end{array}$ & $\begin{array}{l}\quad 15 \\
6 \\
4006 g e \\
\pm \\
\end{array}$ & $\begin{array}{l}10 \\
\quad 7 \\
4256 g f \\
\pm 397\end{array}$ \\
\hline 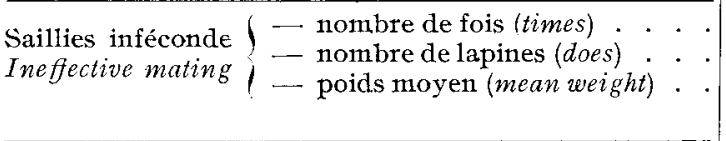 & $\begin{array}{l}19 \\
15 \\
363^{2} g^{d} \\
+269\end{array}$ & $\begin{aligned} & \text { I } 5 \\
& \text { Io } \\
& 4 \text { OI9ge, } n \\
& \pm 345\end{aligned}$ & $\begin{array}{c}6 \\
5 \\
4228 g f 9 h \\
\pm \quad 365\end{array}$ \\
\hline $\begin{array}{l}\text { Saillie fécondante } \\
\text { Fertile mating }\end{array}\left\{\begin{array}{l}\text { - nombre de fois (times) } \\
\text { - nombre de lapines (does) } \\
\text { - poids moyen (mean weight) }\end{array}\right.$ & $\begin{array}{c}9 \\
9 \\
3856 g d, g \\
\pm 27^{2} \\
\end{array}$ & $\begin{aligned} & 19 \\
& 19 \\
& 3970 g^{e, g} \\
& \pm 164 \\
&\end{aligned}$ & $\begin{array}{c}24 \\
24 \\
4255 g f \\
+=366 \\
\end{array}$ \\
\hline 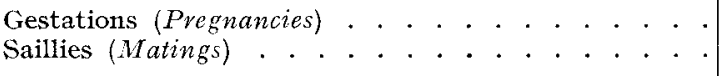 & $9^{9^{i}}$ & $\begin{array}{l}19^{j} \\
34\end{array}$ & $\begin{array}{l}24^{j} \\
3^{\circ}\end{array}$ \\
\hline
\end{tabular}

Les chiffres affectés de la même lettre ne diffèrent pas significativement. There is no significant diff rence between figures dotted with the same letter. 
jusqu'à leur saillie que si on les a rationnées. Mais après un flushing de quelques jours, on peut aussi faire féconder un nombre satisfaisant de lapines. Le rationnement par contre peut empêcher les femelles d'entrer en reproduction.

\section{4. - Performances des premières portées}

Ces données sont fournies à titre indicatif au tableau 4. Les résultats sont médiocres pour les trois groupes. Cette première portée nous pose encore beaucoup de problèmes pour toutes nos jeunes lapines.

TABLEAU 4

Performances des ires portées

Results of the first litters

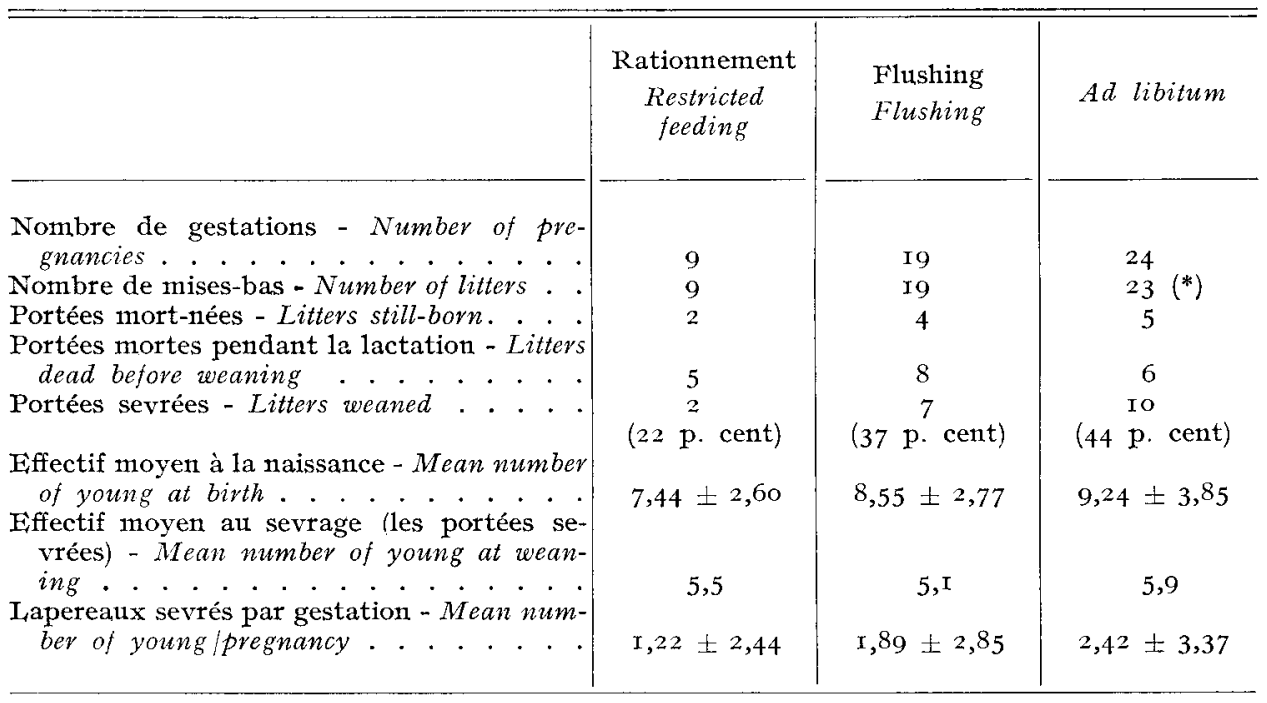

* Une lapine mourait pendant la gestation, deux lapines sont mortes pendant la lactation. One doe died before, and two others after parturition.

\section{5. - Épreuve de fertilité des lapines " $R$ »éliminées}

Les lapines " $\mathrm{R}$ ) éliminées après deux saillies infécondes, ou après cinq refus d'accouplement, sont traitées comme les lapines " $F$ ". Elles ont à ce moment I 8 à 35 jours de plus qu'au début de 1'épreuve. Au total pour les I5 lapines éliminées, en 2 I présentations, I 6 saillies sont obtentres. Onze lapines sont fécondées, dont 9 par la " première " saillie. Le poids corporel a augmenté et est devenu comparable à celui des femelles " $\mathrm{A}$ " à 4 mois. Le poids n'a aucune influence sur le comportement auprès du mâle. De nouveau, les résultats des premières portées sont mauvais. Ces performances sont résumées au tableau 5 . 
TABLEAU 5

Performances des lapines $R$ " infertiles " après un flushing

Results of flushing in "infertile" does

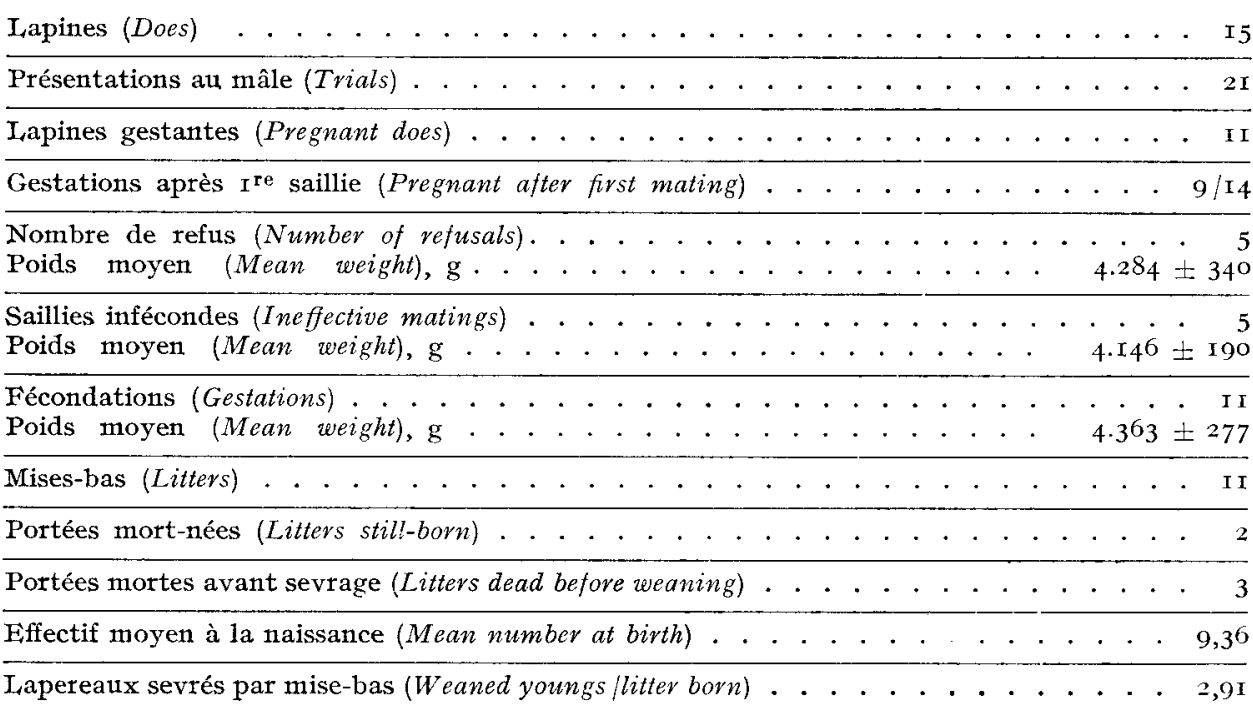

\section{Discussion}

Il faut tout d'abord insister sur le fait que dans cette expérimentation, les lapines alimentées à volonté ont consommé une quantité d'aliment beaucoup plus grande que celle attendue. La cause de cette " hyperphagie " n'est pas déterminée. La croissance rapide poursuivie jusqu'à $4,200 \mathrm{~kg}$ à 4 mois, ne peut expliquer qu'une partie des besoins plus grands. La teneur en énergie de l'aliment, les performances de croissance des lapines utilisées, et la température dans l'élevage suivent les normes recommandées. Un gaspillage d'aliment n'a pas été remarqué lors du contrôle quotidien.

Les lapines sont mises en reproduction dès l'âge de 4 mois. Ce système est employé dans notre cheptel depuis cinq ans au moins. On pourrait dire que les lapines sont trop jeunes à ce moment, bien que les groupes " $\mathrm{F}$ ) et " $\mathrm{A}$ " prouvent leur fertilité à cet âge. La capacité d'allaiter est peut-être plus affectée par cette mise en reproduction hâtive, mais les lapines " $R$ " éliminées, qui sont saillies 3 à 4 semaines plus tard, ne font guère mieux.

Il serait intéressant de suivre ces lapines pendant toute leur vie et de comparer leurs productions totales. Ensuite on pourrait comparer les productions totales de lapines mises en reproduction à différents âges.

Il n'en reste pas moins vrai, que dans notre cheptel expérimental les lapines nullipares ont beaucoup de difficultés à entrer en reproduction lorsqu'elles sont rationnées à ${ }^{5} 5 \mathrm{~g}$ par jour d'un aliment commercial. Elles le font correctement 
lorsqu'elles sont alimentées ad libitum pendant une période de 4 jours au moins avant le premier accouplement, ou lorsqu'elles sont nourries à volonté toute leur vie.

Cette constatation peut être un avertissement pour les éleveurs qui n'emploient pas des races ou des souches sélectionnées, ou qui ont déjà des difficultés avec de jeunes reproductrices.

Un résultat positif de cet essai est le fait que les lapines peuvent être fécondées à 4 mois, et que la période d'alimentation à volonté peut être réduite à 4 jours seulement.

Accepté pour publication en septembre 1977.

\author{
Summary \\ The infuence of three feeding levels of young does on their sexual \\ behaviour and fertility at the age of 4 months
}

After difficulties with respect to the sex drive and the fertility of young does of the breeds "Witte van Dendermonde" and White New Zealand, with continuous feed restriction for nonpregnant rabbits, the hypothesis of insufficient feeding was put forward. Young $f \epsilon$ males weighing about $2.500 \mathrm{~g}$ were selected at the age of in weeks. Out of a group of three sisters the first, " A", was fed without restriction. The second, " $\mathrm{R}$ ", received only I 50 g of feed a day, and the third, " $F$ ", was also restricted but flushed during the week before first mating at 4 months till copulation took place. We had 25 groups. Daily feed-intake of the "A " does was about $300 \mathrm{~g}$ during the raising period and $250 \mathrm{~g}$ during pregnancy.

The results, given by 5 tables, can be summarized as follows. There was a significant difference between the weight of all three groups at 4 months, but at the first fertile mating, weight of restricted and flushed does did not differ any more. The first mating took place earlier in the A does, with 20 pregnancies from 25 matings in 33 trials. Flushed does were mated easily with 14 pregnancies from 24 matings in 33 trials. In restricted does only 5 pregnancies were recorded (20 matings in 65 trials). After 5 trials or 2 matings, for one doe, we got the following results. Group R refused the male 59 times in 87 trials, 19 matings were ineffective and only 9 does became pregnant. Group $\mathrm{F}$ refused $\mathrm{I}_{5}$ times in 49 trials with i 9 ineffective matings and 19 gestations. The A does refused only ro times on 40 trials, only 6 matings were resultless and 24 does became pregnant.

Weaning results of all three groups were bad. All " infer tile " $R$ does were flushed, and then their sex-drive and fertility were comparable with the $F$ group. As a final conclusion of this experiment it seems that severe restriction is not advisable, while restriction followed by flushing might be profit earning. The best results were obtained by does fed ad libitum.

\title{
Références bibliographiques
}

AXEISON J. et ERIKSON S., I953. Energy requirement for maintenance of domestic animals. Kungl. Lantbrukshogskolans Annalev, 20, 5 T-70 (cité par Lebas, I969).

CASIDA L. E., I964. The level of fertility in the female as influenced by feed level and energy intake. Proc. 6th Intern. Congr. Nutrition, Edingbourgh, Aug. r963, p. 366-375.

ÉTIENNE M., DUḰE. P. H., I973. Effects respectifs des niveaux d'alimentation pendant la croissance et le premier mois de gestation sur les performances de reproduction chez la truie nullipare. Résultats préliminaires. Ann. Zootech., 22, 453.

Fitimnne M., HenRy Y., 1973. Influence de l'apport énergétique sur l'utilisation digestive et métabolique des nutriments et les performances chez la truie gestante nullipare. Ann. Zootech., 22, 3 II.

KIRKPATRICK R. L., KIBBE D. J., r971. Restriction and Reproductive Characteristics of captive Cottontail Rabbits. $J$. Wildlive Manag., 35, 2, 332-338. 
GOODE L., WARNick A. C., WALIACE H. P., I965. Effect of dietary energy levels upon reproduction and the relation of endometrial phosphatase activity to embryo survival in gilts. J. Anim. Sci., 24, 959-963.

LAPLACE J. P., LEBAS F., I975. Le transit digestif du lapin III. Influence de 1'heure et du mode d'administration sur l'excrétion fécale du Cérium I $4^{\mathrm{I}}$ chez le lapin alimenté ad libitum, Ann. Zootech., 24, 255-266.

IEATHEM J. H., I966. Nutritional Effects on Hormone Production. J. Anim. Sci., supplément, 68-78.

LEBAS F., I969. L'alimentation du lapin. Extvait du Bulletin de la soc. scientif. d'hyg. alim., 57 (IO-I 2 ), $245^{-268}$.

LEBAS F., 1975a. Besoins nutritifs et Recommandations alimentaires pour le lapin. Contévence à Heveviée, Belgique, pour le "Koninklijke Vlaamse Ingenieuts Vereniging ", 3I-OI-75, 25 pp.

LEBAS F., I975b. Étude chez la lapine de l'influence du niveau d'alimentation durant la gestation. Sur les performances de reproduction. Ann. Zootech., 24, 267-279.

IEBAS F., I975c. Influence de la teneur en énergie de l'aliment sur les performances de croissance chez le lapin. Ann. Zootech., 24, 28I-288.

LEBAS F., LAplace J. P., I974. Mensurations viscérales chez le lapin. III Variations chez la femelle au cours d'un cycle de reproduction en fonction du niveau d'alimentation durant la gestation. Ann. Zootech., 23, 267-292.

LEROX A. M., I949. Normes pour 1'alimentation énergétique. Rapports généraux, 7-80. $5^{\mathrm{C}}$ Congrès de Zootechnie, Paris, 3-Io nov. I949. Impr. RoYer, Paris.

Lodge G. A., Mc. Pherson R. M., I96r. Level of feeding during early life and the subsequent reproductive performance of sows. Anim. Prod, 3, I9-28.

NATIONAI ACADEMY OF SCIENCES, I 966 . Nutrient Requirements of Rabbits. Ist revised edition. National Research Council, Washington D.C.

OKERman F., 1975. Reproduction, Gestion et Techniques d'élevage du Lapin. Conférence à Heverlee, 31-05-75 (Néerl.) I6 pp.

Prud'hon M., Cherubin M., Goussopoulos J., Carles Y., I975. Évolution au cours de la croissance des caractéristiques de la consommation d'aliments solide et liquide du lapin domestique nourri ad libitum. Ann. Zootech., 24, 289-298.

Schieman R., Nehring K., Hoffman L., Jentsch W., Chudy A., I97r. Energetische Futterbewe1tung und Energienormen. Oskar-Kellner-Institut. V E B Deutschev Landwirtschaftsverlag Berlin. 344 pp.

Short R. E., PETers J. B., Casida L. E., r968. Effect of two levels of feeding on ovarian activity, embryo survival and ovarian compensatory hypertrophy in the Rabbit. J. Anim. Sci. 27, 7 OI-704. 